\title{
Ptychographic imaging with a compact gas-discharge plasma extreme ultraviolet light source
}

\author{
M. Odstrcil ${ }^{1,2, *}$, J. Bussmann ${ }^{2,3}$, D. Rudolf 2,3 , R. Bresenitz 2,3 , JiAnWei MiaO ${ }^{4}$, W.S. \\ BROCKLESBY ${ }^{1}$, AND L. JUSCHKIN ${ }^{2,3}$ \\ ${ }^{1}$ Optoelectronics Research Centre, University of Southampton, SO17 1BJ, United Kingdom \\ 2 RWTH Aachen University, Experimental Physics of EUV, JARA-FIT, Steinbachstrasse 15, 52074 Aachen, Germany \\ ${ }^{3}$ Forschungszentrum Jülich GmbH, Peter Grünberg Institut (PGI-9), JARA-FIT, 52425 Jülich, Germany \\ ${ }^{4}$ Department of Physics and Astronomy, and California Nano Systems Institute, University of California, Los Angeles, CA 90095, USA \\ *Corresponding author:mo1e13@soton.ac.uk
}

Compiled October 28, 2015

We report the demonstration of scanning-probe coherent diffractive imaging method (also known as ptychographic CDI) using a compact and partially-coherent gas-discharge plasma source of extreme ultraviolet (EUV) radiation at $17.3 \mathrm{~nm}$ wavelength. Until now, CDI has been mainly carried out with coherent, highbrightness light sources, such as 3rd generation synchrotrons, X-ray free-electron lasers and high harmonic generation. Here we performed ptychographic lensless imaging of an extended sample using a compact, labscale source. The CDI reconstructions were achieved by applying constraint relaxation to the CDI algorithm. Experimental results indicate that our method can handle the low spatial coherence, broadband nature of the EUV illumination as well as the residual background due to visible light emitted by the gas-discharge source. The ability to conduct ptychographic imaging with labscale and partially coherent EUV sources is expected to significantly expand the applications of this powerful CDI method. () 2015 Optical Society of America

OCIS codes: 110.0110 Imaging systems , 040.7480 X-rays, soft $x$-rays, extreme ultraviolet (EUV) ; 110.1650 Coherence imaging; 110.4980 Partial coherence in imaging

http://dx.doi.org/10.1364/ao.XX.XXXXXX

Coherent diffractive imaging (CDI) is a rapidly emerging imaging technique to achieve diffraction-limited resolution without using imaging optics [1-3]. This makes CDI very attractive for imaging in the extreme ultraviolet (EUV) and X-ray spectral range, where the use of focusing optics is limited. In CDI, a coherent wave illuminating a sample produces a diffraction pattern related to the Fourier transform of the sample structure. While the magnitude of the Fourier transform (i.e. the square root of the diffraction intensity) can be collected by a detector, the phase information is lost, which constitutes the wellknown phase problem. If the diffraction intensity is properly measured, the phase information can be retrieved with an iterative algorithm and the sample structure can then be reconstructed [4]. With the rapid development of coherent X-ray sources worldwide, various CDI methods have been demonstrated and have found broad applications in both physical and biological sciences.

One of the powerful CDI methods is termed ptychography (also known as scanning probe CDI) [5], in which an object is scanned relative to a structured illumination probe and a sequence of diffraction patterns is collected with an overlap between adjacent illuminated areas. In contrast to conventional CDI [1-3], ptychography uses the overlapping areas as a real space constraint, allowing the reconstruction of extended objects [5]. For high-resolution imaging, CDI and ptychography experiments typically employ large scale $X$-ray facilities, such as 3rd generation synchrotrons and X-ray free-electron lasers (XFELs) [1-3]. In the last decade, CDI and ptychography have also been successfully implemented with highly coherent tabletop femtosecond lasers generating EUV high harmonics [6-10].

In this letter, we present an example of ptychographic imaging with a partially coherent compact gas-discharge EUV light source operating at $17.3 \mathrm{~nm}$ wavelength (Li-like oxygen, $1 \mathrm{~s}^{2} 2 \mathrm{p}$ $1 \mathrm{~s}^{2} 3 \mathrm{~d}$ transition). In our gas-discharge light source, the emission spectrum in the EUV spectral range is dominated by narrow emission lines of multiply ionized atoms (e.g. $\mathrm{O}^{2+}-\mathrm{O}^{5+}$ ) in a hot dense plasma [11]. The desired wavelength can be selected from various intense and isolated spectral lines. It is possible to change the source spectrum using different gases (e.g. nitrogen, neon, argon and other). Several applications such as EUV lithography [12], EUV and soft X-ray microscopy [13], spectroscopy $[14,15]$ have been demonstrated with similar EUV light sources.

Figure 1 shows the schematic layout of the ptychography experiment. To ensure a sufficient degree of spatial coherence, the plasma radiation was spatially filtered with a $500 \mu \mathrm{m}$ aperture placed $1 \mathrm{~m}$ upstream of the sample. The gas-discharge source along with EUV radiation emits radiation also in UV-visible spectral ranges. $\mathrm{A} \mathrm{Si} / \mathrm{B}_{4} \mathrm{C}$ multilayer Bragg mirror placed at $44^{\circ}$ angle of incidence served as a spectral filter for EUV light with peak reflectivity around $17 \mathrm{~nm}$. However, it is reflective 
for visible light which results in parasitic background (see below). Spectra before and behind the mirror are shown in Fig. 2. The imaged sample was a hexagonal $\mathrm{Si}_{3} \mathrm{~N}_{4}$ grid (Fig. 3(a)). The grid was illuminated through a circular aperture with $10 \mu \mathrm{m}$ in diameter placed less than $100 \mu \mathrm{m}$ away from the sample. The sample was scanned on a $9 \times 10$ regular grid with a step size of $2.5 \mu \mathrm{m}$. A random offset that was $5 \%$ of the step size was implemented to avoid periodic artefacts in the reconstruction [16]. The geometric linear oversampling ratio on the detector was approximately 5 . Figure 3(b) shows an example of the diffraction patterns. The exposure time of each diffraction pattern was $30 \mathrm{~s}$, corresponding to the total number of around $10^{6}$ EUV photons at the sample for each scanning position and 45 minutes of total exposure time.

Along with obvious advantages of compact EUV sources over the large-scale facilities, such as availability and affordable costs, the compact sources have significant drawbacks: low coherent photon flux, lower stability of illumination and lower instrumental alignment precision. This leads to systematic errors (e.g. temporal and spatial incoherence or intensity variations) in the measurements, which usually cannot be removed without significant loss of photon flux. A number of methods have been developed to deal with some of these imperfections [1722], however, usually at the cost of significant computational complexity.

Here, we introduce a linear relaxation method to include a large variation of systematic errors for CDI projection based algorithms, such as temporal and lateral incoherence of the light source as well as background radiation. Standard ptychography method reconstructs the complex-valued object $O(\mathbf{r})$ and the illumination probe $P(\mathbf{r})$ from the collected diffraction patterns $I_{j}$ using the additional information provided by the overlap of the illuminated regions [23]. The illumination probe $P(\mathbf{r})$ is shifted with respect to the sample position by known distances $\mathbf{r}_{\mathbf{j}}=\mathbf{r}-\mathbf{R}_{j}$. In the far-field approximation, the propagated exit-wave $\psi_{j}(\mathbf{k})$ can be expressed using the Fourier transform $\mathfrak{F}$

$$
\psi_{j}(\mathbf{k})=\mathfrak{F}\left(P\left(\mathbf{r}-\mathbf{R}_{j}\right) O(\mathbf{r})\right),
$$

where $\mathbf{k}$ denotes the scattering wave number. The object and illumination probe are assumed to stay constant during the scan. The standard modulus constraint

$$
\tilde{\psi}_{j}(\mathbf{k})=\frac{\sqrt{I_{j}(\mathbf{k})}}{\left|\psi_{j}(\mathbf{k})\right|} \psi_{j}(\mathbf{k})
$$

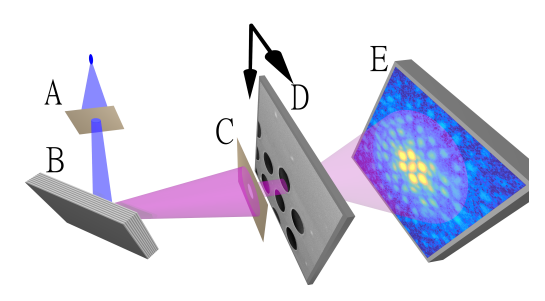

Fig. 1. Schematic layout of our ptychography setup. EUV light from a gas-discharge plasma source is spatially filtered by a $500 \mu \mathrm{m}$ aperture (A) and reflected by a multilayer Bragg mirror (B) to select a wavelength of $17.3 \mathrm{~nm}$. A structured illumination probe defined by a $10 \mu \mathrm{m}$ aperture (C) propagated on the sample (D) that is movable in three dimensions. The diffraction patterns are measured by an EUV sensitive camera (E).

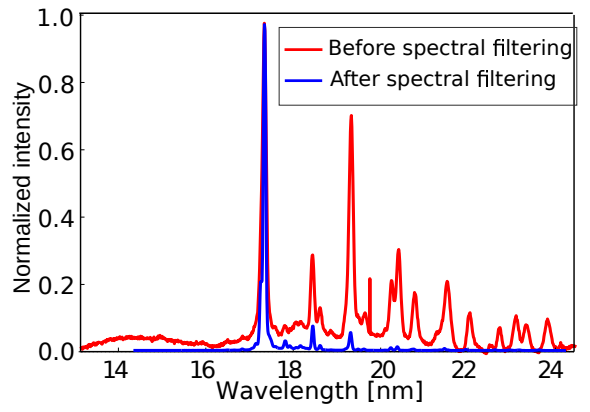

Fig. 2. The spectra of the gas-discharge EUV light source operated with oxygen gas before (red line) and after (blue line) reflection from the multilayer Bragg mirror.
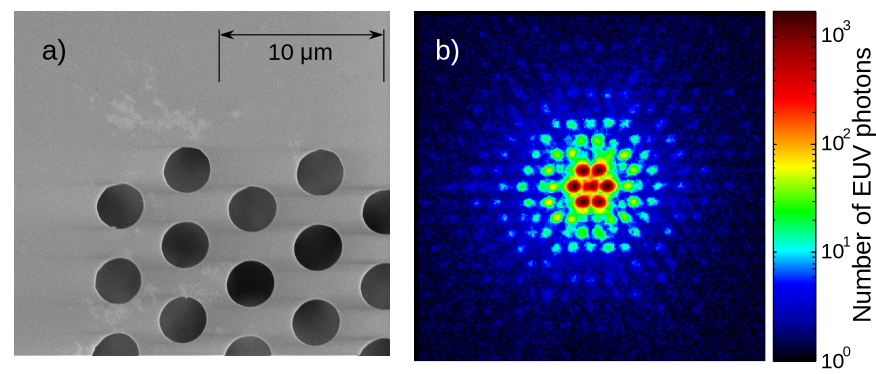

Fig. 3. (a) SEM image of a holey, hexagonal $\mathrm{Si}_{3} \mathrm{~N}_{4}$ grid sample. (b) A representative diffraction pattern $(256 \times 256$ pixels $)$ with an exposure time of $30 \mathrm{~s}$.

is applied in each iteration to replace the magnitude of $\psi_{j}(\mathbf{k})$ with the measured square root intensity values $\sqrt{I_{j}(\mathbf{k})}$ and get an updated estimation $\tilde{\psi}_{j}(\mathbf{k})$ of the propagated exit-wave $\psi_{j}(\mathbf{k})$. The update of the object $O(\mathbf{r})$ and the probe $P(\mathbf{r})$ is dependent on the used projection algorithm (see e.g. Ref. [16, 23]). If the measured diffraction intensity $I_{j}(\mathbf{k})$ cannot be expressed in the form $\left|\psi_{j}(\mathbf{k})\right|^{2}$ (Eq. (1)), e.g. because spatial and temporal coherence requirements are not fulfilled [5], a relaxed version of the modulus constraint is needed. We used a linear relaxation model of the exit wave intensity $\left|\psi_{j}(\mathbf{k})\right|^{2}$ to relax the strict constraints on the data quality

$$
\left|\hat{\psi}_{j}(\mathbf{k})\right|^{2}=\mathbb{A}\left|\psi_{j}(\mathbf{k})\right|^{2}+\mathbf{b}_{j}
$$

where $\mathbb{A}$ is a sparse diagonally dominant matrix and $\mathbf{b}_{j}$ is an positive offset vector. Eq. (3) allows to precalculate the matrix product of multiple corrections and adjust the model by a single sparse matrix multiplication. The noise relaxation can be also applied as shown in Refs. [19, 20].

Generally, the offset vector $\mathbf{b}_{j}$ can be weakly dependent on the reconstruction $\psi_{j}(\mathbf{k})$ and thus slightly differ for each diffraction pattern. In order to construct the relaxation matrix $\mathbb{A}$, it is necessary to rewrite different systematic errors corrections into a matrix forms. In the simplest case of low spatial coherence, the corrected exit-wave model $\left|\hat{\psi}_{j}(\mathbf{k})\right|^{2}$ can be approximated by a convolution with function $g(\mathbf{k})$ that is defined by lateral coherence properties of the illumination [22]

$$
\left|\hat{\psi}_{j}(\mathbf{k})\right|^{2}=\left|\psi_{j}(\mathbf{k})\right|^{2} * g(\mathbf{k}, \sigma)
$$

where $*$ denotes the convolution operator. We have used a Gaussian model [24] of the function $g(\mathbf{k}, \sigma)$ parametrized by $\sigma$. The optimal parameter $\sigma$ is selected to minimize the distance 
between an estimation of the model $\left|\hat{\psi}_{j}(\mathbf{k})\right|^{2}$ and the data $I_{j}$. Equation 4 can be rewritten using the matrix form analogous to the Eq. (3) as a multidiagonal symmetric matrix $\mathbb{A}_{\text {conv }}$ as we show in Code File 1 in the Supplemental materials.

In the case of fully spatially coherent but broadband illumination source, the measured diffraction pattern from an achromatic object can be written as

$$
\left|\psi_{j}^{\text {broad }}(\mathbf{k})\right|^{2}=\int S(\lambda)\left|\psi_{j}(\mathbf{k}, \lambda)\right|^{2} \mathrm{~d} \lambda,
$$

where $S(\lambda)$ denotes the spectral intensity and $\left|\psi_{j}(\mathbf{k}, \lambda)\right|^{2}$ is the monochromatic diffraction pattern with wavelength $\lambda$. Moreover, the intensity of diffraction patterns at different wavelengths $\left|\psi_{j}(\mathbf{k}, \lambda)\right|^{2}$ in the far-field regime is equal to the diffraction pattern at the imaging wavelength $\left|\psi_{j}\left(\mathbf{k}, \lambda_{0}\right)\right|^{2}$ resized by $\lambda / \lambda_{0}$ [21]. Because image rescaling by bilinear interpolation is a linear operation, Eq. (5) can be also written using a sparse matrix $\mathbb{A}_{\text {broad }}$ as we show in Code File 2 in the Supplemental materials. However, if the achromaticity condition is not valid e.g. the spectrum spans across an absorption edge of the sample, full multicolor ptychography needs to be applied [25]. The spectral intensity $S(\lambda)$ can be either measured before experiment by an EUV spectrometer (Fig. 2) or directly in our imaging setup using interferometric spectrometry [15]. The final relaxation matrix $\mathbb{A}$ for a broadband source with low spatial coherence is equal to product of $\mathbb{A}_{\text {broad }}$ and $\mathbb{A}_{\text {conv }}$. Finally, the offset vector $\mathbf{b}_{j}$ accounts for the residual visible light passing through the pinhole and sample. The broadband visible light background (Fig. 4(b)) passes through the same aperture (see Fig. 1(A)) as the EUV light, and therefore, it is spatially highly coherent. The visible light scattering affects mainly the high spatial frequencies of the diffraction pattern, where the diffracted EUV signal is low compared to the scattered visible light. We propose a method to identify $\mathbf{b}_{j}$ based on a robust iterative filtering. While methods for static or flat background correction were already presented in Ref. [20, 26], our method is designed to remove variable background with the main assumption of sufficient spectral separation between the imaging wavelength $\lambda_{0}=17.3 \mathrm{~nm}$ and the visible light background pattern. Figure 4(b) shows the measured visible light spectrum $S_{\text {vis }}(\lambda)$, which is used to calculate an average probe $P_{\text {vis }}(\mathbf{r})$ for the visible light according to the equation

$$
P_{\text {vis }}(\mathbf{r})=\int\left|S_{\text {vis }}(\lambda) P(\lambda, \mathbf{r})\right| \mathrm{d} \lambda
$$

where $P(\lambda, \mathbf{r})$ denotes the illumination probe resized by a factor of $\lambda_{0} / \lambda$. The background can be estimated using

$$
\mathbf{b}_{j}=\mathfrak{F}^{-1}\left[\mathfrak{F}\left(I_{j}-\left|\psi_{j}\right|^{2}\right) \frac{P_{\text {vis }}}{\max \left(P_{\text {vis }}\right)}\right]
$$

where normalized $P_{\text {vis }}(\mathbf{r})$ acts as a frequency filter. For practical application, a robust version of Eq. (7) needs to be applied because of large misfits in the low spatial frequencies during convergence of the CDI algorithm. The background estimation in $\mathrm{n}$-th iteration is expressed as

$$
\begin{array}{r}
\mathbf{b}_{j}^{n+1}=\alpha \mid \mathfrak{F}^{-1}\left[\mathfrak { F } \left(W_{n}\left(I_{j}-\left|\psi_{j}\right|^{2}-\mathbf{b}_{j}^{n}\right)\right.\right. \\
\left.\left.+\mathbf{b}_{j}^{n}\right) \frac{P_{\mathrm{vis}}}{\max P_{\mathrm{vis}}}\right] \mid \\
+(1-\alpha) \mathbf{b}_{j}^{n}
\end{array}
$$

where $\alpha=1.75$ is used as a relaxation constant to speed up the convergence, and $W_{n}$ (between 0 and 1 ) is a robust weighting vector calculated using the Tukey's bisquare function [27].
To demonstrate the advantages of the linear relaxation method, we compared the ptychographic reconstruction using the standard ePIE algorithm (Fig. 5(a)) to that using the linear correction (Fig. 5(b)). Figure 5(a) shows many artifacts due to the background and partial coherence of the illumination probe. These artifacts are removed after combining the linear correction method with the ePIE algorithm (Fig. 5(b)). Note that there was no improvement if the lateral incoherence relaxation (Eq. (4)) was replaced by the state mixtures method [18] because the assumtion of the Gaussian incoherence model was sufficiently precise for our EUV illumination as it was shown in Ref. [24].

The pixel size was estimated from the experimental geometry to $223 \mathrm{~nm}$ and then more precisely refined during the ptychographic reconstruction by a cross-correlation-based method [28] to $228 \mathrm{~nm}$. The spatial half-pitch resolution of $255 \pm 10 \mathrm{~nm}$ was estimated by a sigmoidal fit across the hole edges using the $10 \%$ to $90 \%$ criterion [6]. We estimated the optimal incoherence width $\sigma$ of the kernel $g$ (Eq. (4)) to be 1.67 pixel, which corresponds to a lateral coherence length of about $14 \mu \mathrm{m}$. Furthermore, using the refractive index of $\mathrm{Si}_{3} \mathrm{~N}_{4}$ at $17.3 \mathrm{~nm}$ [29], we estimated the grid thickness from the phase and amplitude reconstruction to be $172 \pm 5 \mathrm{~nm}$ (Fig. 5(c)), which compares well to $182 \pm 5 \mathrm{~nm}$ thickness measured by scanning electron microscopy. The reconstructed average visible light background intensity at different scanning positions (average of entries of the vector $\mathbf{b}_{j}$ ) from Eq. (3) is shown in Fig 4(a). In the upper right corner, which corresponds to the position of the holes in the $\mathrm{Si}_{3} \mathrm{~N}_{4}$ grid (Fig. 5), the background is reduced because a larger fraction of the visible light is scattered out of the detector area. Figure 4(b) shows the measured visible light spectrum, which contains many spectral lines around $400 \mathrm{~nm}$. In principle, visible light can be blocked by a thin EUV light transmitting filter, such as an aluminum foil. However, such filters also absorb a significant fraction of the EUV light, while our background removal method allows the use of the full EUV intensity.

In summary, we have demonstrated ptychographic imaging with a partially coherent compact gas-discharge EUV light source at $17.3 \mathrm{~nm}$ wavelength with a lateral resolution of $255 \mathrm{~nm}$ and 30 s exposure time at each scanning position resulting in 45 minutes of total exposure time. A linear relaxation model was introduced to elegantly include a large number of corrections to any projection-based CDI method without significant increase of computation time per iteration. In the future experiments, curved illumination wavefront will be applied in order to reduce the incoherence effects and dynamic range of the collected diffraction patterns. In combination with another fuel material, e.g. Xe and higher reflexivity EUV mirrors, we estimate that the useful photon flux for CDI can be increased by 2-4 orders of magnitude and enable CDI at $13 \mathrm{~nm}$ wavelength. A possible application of our imaging setup is inspection of multilayer mask blanks for the EUV lithography.

M.O. acknowledges financial support from the EU FP7 Erasmus Mundus Joint Doctorate Programme EXTATIC under framework partnership agreement FPA-2012-0033. L.J. acknowledges financial support by the Helmholtz Association for a Helmholtz Professorship as a part of the Initiative and Networking Fund and J.M. thanks for the support by the DARPA PULSE program through a grant from AMRDEC (DARPABAA-12-63). The data for this work is accessible through the University of Southampton Institutional Research Repository (DOI:10.5258/SOTON/382965) 

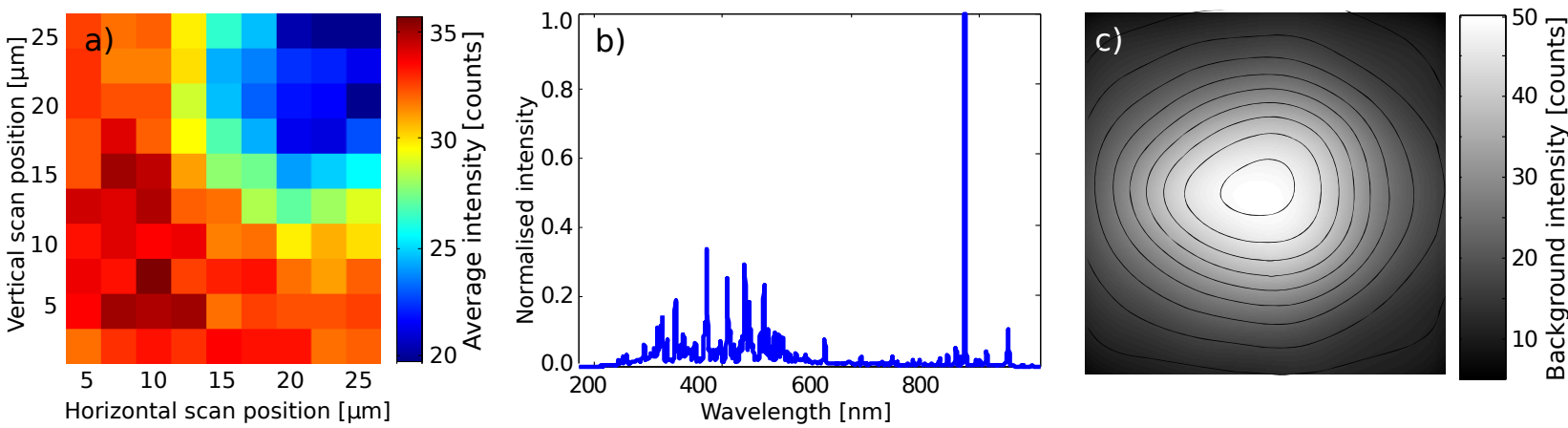

Fig. 4. (a) Average background intensity ( $\mathbf{b}_{j}$ from Eq. (3)) determined by our background correction method for each scanning position $j$. (b) Measured spectrum of the gas-discharge light source in the visible spectral range. (c) One example of the removed visible light background pattern.
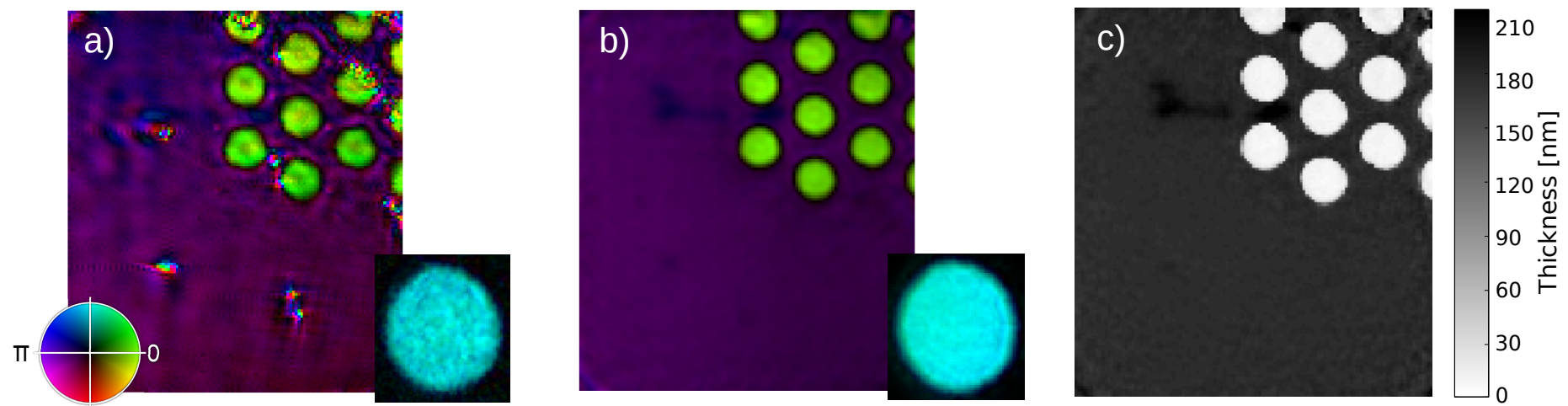

Fig. 5. Comparison between the reconstruction of the object and the probe (inset) with the standard ePIE algorithm (a) and with our improved method using linear relaxation model (b). Both images are displayed in the same intensity range. The magnitude and phase of the reconstruction are encoded using the hue-saturation-value scheme. (c) Thickness of the $\mathrm{Si}_{3} \mathrm{~N}_{4}$ layer was calculated from the reconstructed phase. Compared to (a), the features are sharper and artifacts are reduced in the corrected image.

\section{REFERENCES}

1. J. Miao, T. Ishikawa, I. K. Robinson, and M. M. Murnane, Science 348, 530 (2015).

2. K. A. Nugent, Adv. Phys 59, 1 (2010).

3. P. Thibault and V. Elser, Annu. Rev. Cond. Mat. Phys 1 (2010).

4. J. Miao, P. Charalambous, J. Kirz, and D. Sayre, Nature 400, 342 (1999).

5. J. Rodenburg, Adv. Imag. Elec. Phys. 150, 87 (2008).

6. M. D. Seaberg, D. E. Adams, E. L. Townsend, D. a. Raymondson, W. F. Schlotter, Y. Liu, C. S. Menoni, L. Rong, C.-C. Chen, J. Miao, H. C. Kapteyn, and M. M. Murnane, Opt. Express 19, 22470 (2011).

7. M. D. Seaberg, B. Zhang, D. F. Gardner, E. R. Shanblatt, M. M. Murnane, H. C. Kapteyn, and D. E. Adams, Optica 1, 39 (2014).

8. R. L. Sandberg, A. Paul, D. A. Raymondson, S. Hädrich, D. M. Gaudiosi, J. Holtsnider, I. T. Raanan, O. Cohen, M. M. Murnane, H. C. Kapteyn, C. Song, J. Miao, Y. Liu, and F. Salmassi, Phys. Rev. Lett. 99, 098103 (2007).

9. A. Parsons, R. Chapman, P. Baksh, B. Mills, S. Bajt, W. Brocklesby, and J. Frey, J. Opt. 15, 094009 (2013).

10. H. V. Le, K. B. Dinh, P. Hannaford, and L. Van Dao, J. Appl. Phys 116, 173104 (2014).

11. M. Benk and K. Bergmann, J. Micro/Nanolith. 11, 1 (2012).

12. S. Danylyuk, H.-s. Kim, S. Brose, C. Dittberner, P. Loosen, T. Taubner, K. Bergmann, and L. Juschkin, Vac. Sci. Technol. B 31, 021602 (2013).

13. L. Juschkin, R. Freiberger, and K. Bergmann, in "J. Phys., Conf. Ser.", , vol. 186 (2009), vol. 186, p. 012030.

14. D. Wilson, D. Rudolf, C. Weier, R. Adam, G. Winkler, R. Frömter, S. Danylyuk, K. Bergmann, D. Grützmacher, C. M. Schneider, and
L. Juschkin, Rev. Sci. Instrum. 85, 103110 (2014).

15. D. Rudolf, J. Bußmann, M. Odstrčil, M. Dong, K. Bergmann, S. Danylyuk, and L. Juschkin, Opt. Lett. 40, 2818 (2015).

16. P. Thibault, M. Dierolf, O. Bunk, A. Menzel, and F. Pfeiffer, Ultramicroscopy 109, 338 (2009).

17. B. Enders, M. Dierolf, P. Cloetens, M. Stockmar, F. Pfeiffer, and P. Thibault, Appl. Phys. Lett. 104, 171104 (2014).

18. P. Thibault and A. Menzel, Nature 494, 68 (2013).

19. P. Thibault and M. Guizar-Sicairos, New Jour. of Phys. 14 (2012).

20. S. Marchesini, A. Schirotzek, C. Yang, H.-t. Wu, and F. Maia, Inverse Problems 29 (2013).

21. B. Abbey, L. W. Whitehead, H. M. Quiney, D. J. Vine, G. A. Cadenazzi, C. A. Henderson, K. A. Nugent, E. Balaur, C. T. Putkunz, A. G. Peele, G. J. Williams, and I. McNulty, Nature Photonics 5, 420 (2011).

22. J. N. Clark and A. G. Peele, Appl. Phys. Lett. 99, 154103 (2011).

23. A. M. Maiden and J. M. Rodenburg, Ultramicroscopy 109, 1256 (2009).

24. J. Bußmann, M. Odstrčil, R. Bresenitz, D. Rudolf, J. Miao, W. S. Brocklesby, D. Grutzmacher, and L. Juschkin, Proc. SPIE 9589, 95890L-8 (2015).

25. D. J. Batey, D. Claus, and J. M. Rodenburg, Ultramicroscopy 138 (2014).

26. S. T. Thurman and J. R. Fienup, JOSA A 26 (2009).

27. R. Maronna, D. Martin, and V. Yohai, Robust statistics (2006).

28. F. Zhang, I. Peterson, J. Vila-comamala, A. Diaz, R. Bean, B. Chen, A. Menzel, I. K. Robinson, and J. M. Rodenburg, Opt. Express 21, 13592 (2013).

29. B. L. Henke, E. M. Gullikson, and J. C. Davis, Atomic Data and Nuclear Data Tables 54, 181 (1993). 


\section{REFERENCES}

1. J. Miao, T. Ishikawa, I. K. Robinson, and M. M. Murnane, "Beyond crystallography: Diffractive imaging using coherent $x$-ray light sources," Science 348, 530-535 (2015).

2. K. A. Nugent, "Coherent methods in the x-ray sciences," Advances in Physics 59, 1-99 (2010).

3. P. Thibault and V. Elser, "X-ray diffraction microscopy," Condensed Matter Physics 1 (2010).

4. J. Miao, P. Charalambous, J. Kirz, and D. Sayre, "Extending the methodology of $x$-ray crystallography to allow imaging of micrometresized non-crystalline specimens," Nature 400, 342-344 (1999).

5. J. Rodenburg, "Ptychography and related diffractive imaging methods," Advances in Imaging and Electron Physics 150, 87-184 (2008).

6. M. D. Seaberg, D. E. Adams, E. L. Townsend, D. A. Raymondson, W. F. Schlotter, Y. Liu, C. S. Menoni, L. Rong, C.-C. Chen, J. Miao et al., "Ultrahigh $22 \mathrm{~nm}$ resolution coherent diffractive imaging using a desktop 13 nm high harmonic source," Optics Express 19, 2247022479 (2011).

7. M. D. Seaberg, B. Zhang, D. F. Gardner, E. R. Shanblatt, M. M. Murnane, H. C. Kapteyn, and D. E. Adams, "Tabletop nanometer extreme ultraviolet imaging in an extended reflection mode using coherent fresnel ptychography," Optica 1, 39-44 (2014).

8. R. L. Sandberg, A. Paul, D. A. Raymondson, S. Hädrich, D. M. Gaudiosi, J. Holtsnider, I. T. Raanan, O. Cohen, M. M. Murnane, H. C. Kapteyn et al., "Lensless diffractive imaging using tabletop coherent high-harmonic soft-x-ray beams," Physical Review Letters 99, 098103 (2007).

9. A. Parsons, R. Chapman, P. Baksh, B. Mills, S. Bajt, W. Brocklesby, and J. Frey, "Ultra-broadband support determination for extreme ultraviolet coherent diffractive imaging from a high harmonic source," Journal of Optics 15, 094009 (2013).

10. H. V. Le, K. B. Dinh, P. Hannaford, and L. Van Dao, "High resolution coherent diffractive imaging with a table-top extreme ultraviolet source," Journal of Applied Physics 116, 173104 (2014).

11. M. Benk and K. Bergmann, "Brilliance scaling of discharge sources for extreme-ultraviolet and soft x-ray radiation for metrology applications," Journal of Micro/Nanolithography, MEMS, and MOEMS 11, 021106-1 (2012).

12. S. Danylyuk, H.-s. Kim, S. Brose, C. Dittberner, P. Loosen, T. Taubner, K. Bergmann, and L. Juschkin, "Diffraction-assisted extreme ultraviolet proximity lithography for fabrication of nanophotonic arrays," Journal of Vacuum Science \& Technology B 31, 021602 (2013).

13. L. Juschkin, R. Freiberger, and K. Bergmann, "Euv microscopy for defect inspection by dark-field mapping and zone plate zooming," in "Journal of Physics: Conference Series," , vol. 186 (IOP Publishing, 2009), vol. 186, p. 012030.

14. D. Wilson, D. Rudolf, C. Weier, R. Adam, G. Winkler, R. Frömter, S. Danylyuk, K. Bergmann, D. Grützmacher, C. M. Schneider et al., "Generation of circularly polarized radiation from a compact plasmabased extreme ultraviolet light source for tabletop x-ray magnetic circular dichroism studies," Review of Scientific Instruments 85, 103110 (2014).

15. D. Rudolf, J. Bußmann, M. Odstrčil, M. Dong, K. Bergmann, S. Danylyuk, and L. Juschkin, "Interferometric broadband fourier spectroscopy with a partially coherent gas-discharge extreme ultraviolet light source," Optics Letters 40, 2818-2821 (2015).

16. P. Thibault, M. Dierolf, O. Bunk, A. Menzel, and F. Pfeiffer, "Probe retrieval in ptychographic coherent diffractive imaging," Ultramicroscopy 109, 338-343 (2009).

17. B. Enders, M. Dierolf, P. Cloetens, M. Stockmar, F. Pfeiffer, and P. Thibault, "Ptychography with broad-bandwidth radiation," Applied Physics Letters 104, 171104 (2014).

18. P. Thibault and A. Menzel, "Reconstructing state mixtures from diffraction measurements," Nature 494, 68-71 (2013).

19. P. Thibault and M. Guizar-Sicairos, "Maximum-likelihood refinement for coherent diffractive imaging," New Journal of Physics 14, 063004 (2012)

20. S. Marchesini, A. Schirotzek, C. Yang, H.-t. Wu, and F. Maia, "Aug- mented projections for ptychographic imaging," Inverse Problems 29 115009 (2013).

21. B. Abbey, L. W. Whitehead, H. M. Quiney, D. J. Vine, G. A. Cadenazzi, C. A. Henderson, K. A. Nugent, E. Balaur, C. T. Putkunz, A. G. Peele et al., "Lensless imaging using broadband x-ray sources," Nature Photonics 5, 420-424 (2011).

22. J. N. Clark and A. G. Peele, "Simultaneous sample and spatial coherence characterisation using diffractive imaging," Applied Physics Letters 99, 154103 (2011).

23. A. M. Maiden and J. M. Rodenburg, "An improved ptychographical phase retrieval algorithm for diffractive imaging," Ultramicroscopy 109 1256-1262 (2009)

24. J. Bußmann, M. Odstrcil, R. Bresenitz, D. Rudolf, J. Miao, W. S. Brocklesby, D. Grutzmacher, and L. Juschkin, "Employing partially coherent, compact gas-discharge sources for coherent diffractive imaging with extreme ultraviolet light," Proc. SPIE 9589, 95890L-8 (2015).

25. D. J. Batey, D. Claus, and J. M. Rodenburg, "Information multiplexing in ptychography," Ultramicroscopy 138, 13-21 (2014).

26. S. T. Thurman and J. R. Fienup, "Phase retrieval with signal bias," JOSA A 26, 1008-1014 (2009).

27. R. Maronna, D. Martin, and V. Yohai, Robust statistics (John Wiley \& Sons, Chichester. ISBN, 2006).

28. F. Zhang, I. Peterson, J. Vila-Comamala, A. Diaz, F. Berenguer, R. Bean, B. Chen, A. Menzel, I. K. Robinson, and J. M. Rodenburg, "Translation position determination in ptychographic coherent diffraction imaging," Optics Express 21, 13592-13606 (2013).

29. B. L. Henke, E. M. Gullikson, and J. C. Davis, "X-ray interactions: photoabsorption, scattering, transmission, and reflection at $\mathrm{e}=50-30,000$ ev, z= 1-92," Atomic data and nuclear data tables 54, 181-342 (1993). 